\title{
Dynamic view of critical state regime in granular materials: a mesoscale per- spective
}

\author{
Na Deng ${ }^{1, *}$, Antoine Wautier ${ }^{2}$, Yannick Thiery ${ }^{3}$, Zhen-Yu Yin ${ }^{4}$, Pierre-Yves Hicher ${ }^{5}$, and François Nicot $^{1}$ \\ ${ }^{1}$ Université Grenoble Alpes, INRAE, UR ETGR, 2 rue de la Papeterie-BP 76, 38402 St-Martin-d'Heres, France \\ ${ }^{2}$ INRAE, Aix-Marseille University, UR RECOVER, 3275 Rte Cézanne, CS 40061, 13182 Aix-en-Provence Cedex 5, France \\ ${ }^{3}$ BRGM (French Geological Survey), Risk and Prevention Division, Orléans, France \\ ${ }^{4}$ Department of Civil and Environmental Engineering, the Hong Kong Polytechnic University, Hong Kong, China \\ ${ }^{5}$ Research Institute of Civil Engineering and Mechanics (GeM), UMR CNRS 6183, Ecole Centrale de Nantes, Nantes, France
}

\begin{abstract}
We revisit the Critical State (CS) in a dynamic perspective at a mesoscopic scale to enrich the classical view of the critical state as a steady state. The dynamics of meso-structures has been characterized by recording generating and vanishing processes in discrete element simulations. Our results suggest that mesostructures have a short but regular lifespan at critical state along a biaxial compression loading path.
\end{abstract}

\section{Introduction}

Critical state refers to a stationary state where stress and volume of geomaterials remain constant under continuous shear strain. As an essential reference state in constitutive models based on the Critical State Theory (CST) [1, 8] or the more recent Anisotropic Critical State Theory (ACST) [3], the bulk stationarity at CS has been received acute attention from both macroscopic and microscopic scales. However, critical state stems from local rearrangement of particles under loading. From this perspective, structures at critical states, even statistically stationary, are in dynamic evolution.

A variety of theories or indexes were introduced to characterize particle rearrangement at mesoscale (i.e. at the scale of a few grains), and to extract some elementary mechanisms responsible for the complex macroscopic behaviour. In relation with critical state, Tordesillas [4] have proposed the jamming-unjamming theory (which was initially proposed by [5]) to explain the energy flow in deforming granular systems. The origin of jamming has been attributed to force chains, and is associated with solid-like behaviour. Unjamming transition is a state in which contacts become mobilized via rolling or sliding. When the unjamming and jamming reach a balance during loading, the critical state is obtained. In the present study, we propose to characterize quantitatively these dynamic processes before and after the balance is reached by considering force chains and grain loops (two important meso-structure descriptors of granular materials) and introducing the concepts of lifespan and life expectancy.

The importance of investigations on the dynamic microstructure evolutions at CS is twofold: (1) providing a complementary view to the classical view of statistical sta-

*e-mail: na.deng@inrae.fr

A video is available at https://doi.org/10.48448/zfqx-ra44 tionarity of the micro-structure at critical state, (2) helping to find clues to enrich micromechanical constitutive models (predicting macroscopic stress-strain behaviour directly and explicitly based on micro and mesoscopic structures) and make them able to recover the critical state directly from mesoscale considerations. The paper is organized as follows. In section 2 , we review the mechanical response along a biaxial test using discrete element method (DEM). Emphasis will be given to dynamic evolution from a mesoscale perspective in Section 3. We conclude in Section 4.

\section{DEM simulation and results}

\subsection{Simulation program}

A conventional biaxial test is carried out numerically using the open source software YADE [9]. Under consideration here is a $2 \mathrm{D}$ soil sample in the form of an assembly of a single layer of 20,000 spherical particles contained within a domain of $1 \mathrm{~m} \times 1.5 \mathrm{~m}$. The particle sizes have an average diameter $d_{50}=0.008 \mathrm{~m}$ and $d_{\max } / d_{\min }=2$. The contact is described by a linear elasto-frictional law. The normal and tangential linear spring of respective stiffness $k_{n}$ and $k_{t}$ are characterized by $k_{n} / D_{s}$ being $300 \mathrm{MPa}$, where $D_{s}=2 R_{1} R_{2} /\left(R_{1}+R_{2}\right)$ and $R_{1}, R_{2}$ are the radii of particles in a given contact, and $k_{t} / k_{n}$ being 0.5 . A sliding limit characterized by a friction angle $\phi=35^{\circ}$. The boundary conditions are shown in Fig. 1. The numerical model has been used in previous paper and more details about it can be found in $[2,6]$. The selected material parameters are quite standard for dry cohesionless granular materials and the following results should remain qualitatively unchanged for different sets of parameters corresponding to realistic granular materials. 


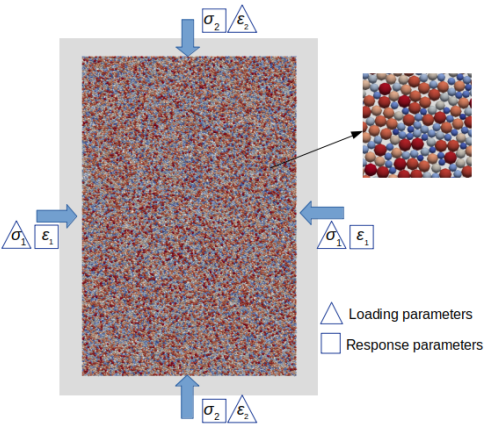

Figure 1. 2D DEM specimen for a biaxial test where loading parameters are $\varepsilon_{1}$ and $\sigma_{2}$

\subsection{Stress-strain analysis}

The stress and strain states are described in 2D as follows: deviatoric stress $q=\sigma_{2}-\sigma_{1}$ and volumetric strain $\varepsilon_{v}=\varepsilon_{1}+\varepsilon_{2}$, where $\sigma_{1}$ and $\sigma_{2}$ are the principal stresses at horizontal and vertical directions and $\varepsilon_{1}$ and $\varepsilon_{2}$ are the principal strains, as shown in Fig. 1. Macroscopic responses of the tested specimens are illustrated in Fig. 2, in which deviatoric stress and volumetric strain evolve with respect to the axial strain. The figure shows typical stress and strain responses of a dense sample under the biaxial loading. It can be observed that at critical states, both $q$ and $\varepsilon_{v}$ fluctuate around a constant value. The fluctuations are paid less attention besides the research of [10] who analysed the force chains buckling. Based on this result, the dynamic equilibrium of CS is investigated in the following section.

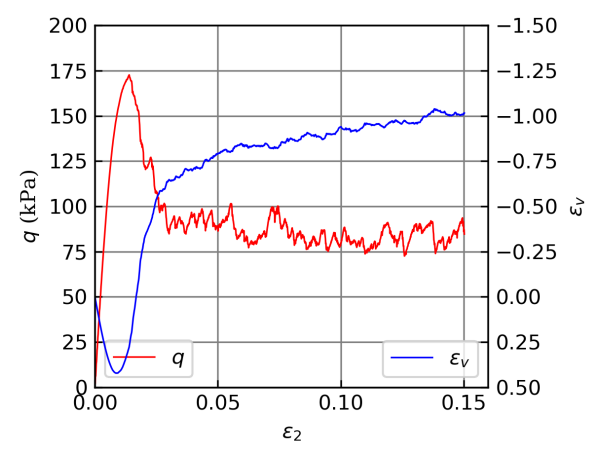

Figure 2. The evolution of deviatoric stress and volumetric strain with respect to the axial strain

\section{Dynamics at critical state}

As highlighted in Introduction, the jamming and unjamming behaviour at CS includes dynamic mechanism at mesoscale. In a similar spirit, the rearrangement of particles along the simulation has been characterized by recording generating and vanishing processes of chained particles (i.e. particles belonging to force chains) and grain loops. As shown in Fig. 3, three conditions should be satisfied for a force chain: more than 3 particles, the principal stress of each particle $\sigma_{p}$ being larger than the averaged assembly stress, the relative orientation between contact and principal stress direction $\theta$ being less than $45^{\circ}$. Grain loops, enclosed by contact branches, are categorized into four groups according to the side number [2, 7, 11]. Note that the wording generating/vanishing relates to incremental evolutions of the sets of chained particles and grain loops during DEM simulations for axial strain increments $\Delta \varepsilon_{2}=0.1 \%$.

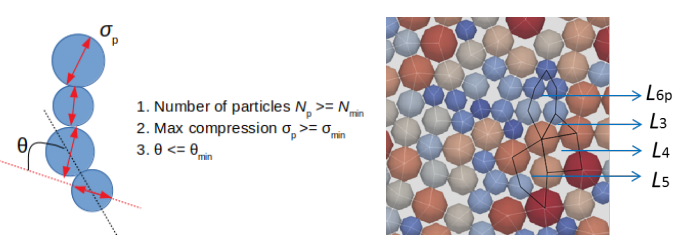

Figure 3. Force chain (left) and grain loop (right)

\section{Dynamics at critical state}

\subsection{Generating and vanishing process of chained particles}

All chained particles are tracked from birth, i.e. the first time they become part of a given force chain, to death, i.e. the first time they no longer belong to any force chain. The lifespan and life expectancy of each chained particle can be characterized by how much time (characterized by incremental axial strain) the particle has been existing as a chained particle without interruption before and after the reference state, respectively. This enables to construct the probability $\left(P_{\mathrm{g} / \mathrm{v}}\right)$ for the lifespan and the life expectancy of chained particles at any time of the simulation, which also corresponds to the generation and vanishing process of the chained particles.

In Fig. 4, the probability for life expectancy are displayed in red for the initial state and two critical states, while probability for lifespan are displayed in blue for the same two critical states. Because the sample is known to experience shear band localization (not shown here), note that the analysis is restricted to particles belonging to the shear band domain in Fig. 4 (II).

In Fig. 4 (I), it can be observed that a part of the chained particles existing at the initial state disappears during the loading before stress stabilizes, while nearly $30 \%$ remains permanently within the force chains until the end of the test, most of them located outside of the shear band domain. The vanishing process can be divided into three stages according to the curve giving the rate of transition from chained particles to non-chained particles: a rapid drop at the stage C.I, a gentle decrease at the stage C.II and nearly no change at the stage C.III. Capital C represents here the chained particles.

The transition point between the stage C.I and the stage C.II corresponds exactly to the characteristic point, which corresponds to the equality between incremental elastic strain energy and incremental plastic strain energy [11]. The decrease in the chained particle $P_{\mathrm{g} / \mathrm{v}}$, namely the 
vanishing of chained particles existing at the initial state can be explained as follows. Under a vertical load, the assembly starts to build up a vertical force chain network. This network will not live long as the lateral dilatation of the sample imposed by the biaxial loading increasingly opens the lateral contacts supporting the force chains. At the characteristic point, the plasticity mechanisms become dominant, force chains bend and collapse, which result in a gentle decrease in the force chain $P_{\mathrm{g} / \mathrm{v}}$. The stage C.III corresponds to the CS characterized by a constant deviatoric stress and a constant volumetric strain. It demonstrates that once the sample reaches the CS, chained particles developed in the initial state nearly stop disappearing; the remaining ones belong indeed to the sample domain outside the shear band.

At the state $\mathrm{M}$ (resp. N) in Fig. 4 (II), the $P_{\mathrm{g} / \mathrm{v}}$ before and after the state $\mathrm{M}$ (resp. $\mathrm{N}$ ) demonstrate the generating and vanishing processes of chained particles within the shear band domain. Few chained particles existing at $\mathrm{M}$ or $\mathrm{N}$ come from the initial state, which proves that the initial arrangement of particles has been erased when the sample reaches CS. The symmetric shape of the lifespan and life expectancy $P_{\mathrm{g} / \mathrm{v}}$ shows that the rearrangement of chained particles reaches an equilibrium at CS. More interestingly the shape of the $P_{\mathrm{g} / \mathrm{v}}$ for the states $\mathrm{N}$ and $\mathrm{M}$ is almost the same. Force chains are always rearranging at critical state and the rate of renewal of the set of chained particles is constant.

Furthermore, it can be seen in Fig. 4 (I) that nearly $30 \%$ of the chained particles in the whole sample remain chained from the beginning to the end of the test. On the contrary, a small number of chained particles present within the shear band at critical state were generated at the initial state (Fig. 4 (II)). Thus, the chained particles remaining alive throughout the whole loading process belong almost all to the domain outside the shear band.

\subsection{Generating and vanishing process of grain loops}

To further understand the critical state dynamics, the generating and vanishing of grain loops are analysed in Fig. 5 for loops composed of 3, 4, 5 and 6 or more particles. Lifespan and life expectancy probabilities are computed for the initial state considering the whole sample (I) and two critical states within the shear band (II). Observations similar to those which have been reported in the previous subsection can be found in Fig. 5: (1) around $80 \%$ of the loops existing at the initial state vanish gradually during the early stage of the loading; (2) the symmetrical shape of the $P_{\mathrm{g} / \mathrm{v}}$ at CS suggests that the generating and vanishing processes share strong common features and demonstrate that the rearrangement of loops reaches an equilibrium at CS.

Based on Figures 4 and 5, a novel way for understanding CS from the perspective of a mesoscale dynamics can be inferred: the critical state results from the generating and vanishing of meso-clusters until a dynamic equilibrium is reached as shown by the symmetry of the probabilities for
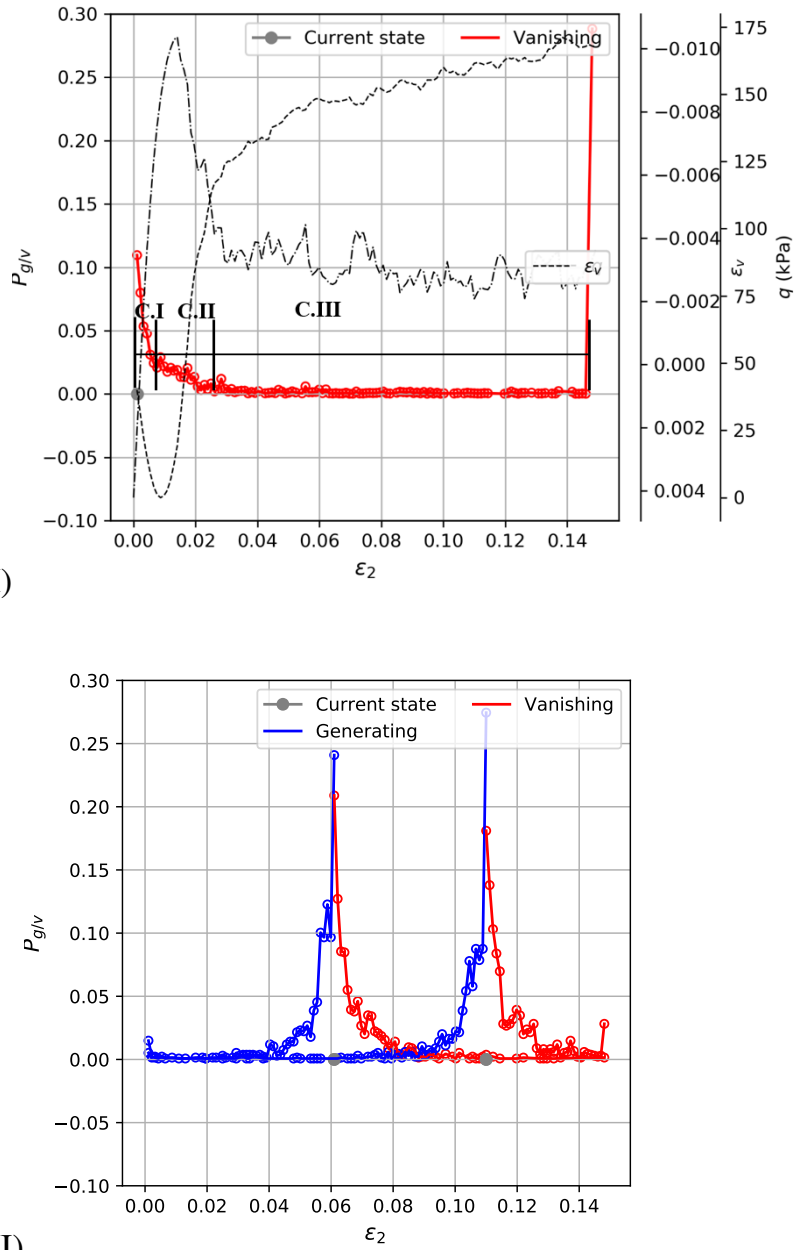

Figure 4. Lifespan and life expectancy probabilities of chained particles at the initial state considering the whole sample (I) and two critical states within the shear band (II).

lifespan and life expectancy.

\section{Conclusions}

In the previous work [4, 10], CS appears to be governed by the competing mechanisms of growth and collapse of force chains. Consistently, we have shown that the critical state results from the generating and vanishing of meso-clusters at similar rates. We have established that force chains and grain loops have a short life at critical state, corresponding to a rapid fading of the material memory to its loading history. These new findings might help to find clues to incorporate explicitly CS features in micro-mechanical constitutive models.

Most newly formed chained particles and grain loops are located within the shear bands and these newly formed chained particles and grain loops die out quickly. They coexist with many old, robust chained particles and grain loops, mainly located outside the shear bands. 

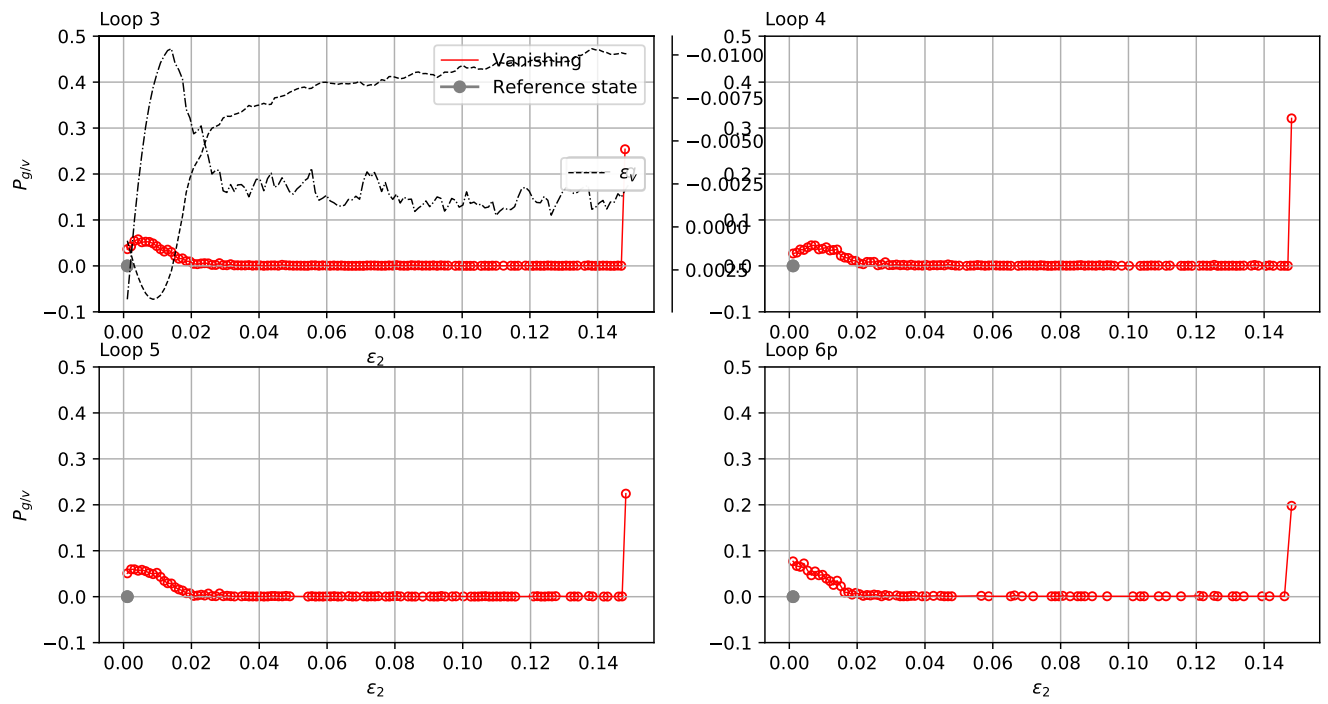

(I)


(II)

Figure 5. Lifespan and life expectancy probabilities of grain loops at the initial state considering the whole sample (I) and two critical states within the shear band (II).

\section{References}

[1] A. Casagrande, J. Boston Society of Civil Engineers 23, 13 (1936)

[2] N. Deng, A. Wautier, Y. Thiery, Z. Y. Yin, P. Y. Hicher, F. Nicot, J Mech Phys Solids 149, 104300 (2021)

[3] X. S. Li, Y. F. Dafalias, J. Eng. Mech. 138, 263 (2012)

[4] A. Tordesillas, Philos Mag 87, 4987 (2007)

[5] A. J. Liu, S. R. Nagel, Nature 396, 21 (1998)

[6] J. Liu, A. Wautier, S. Bonelli, F. Nicot, F. Darve, Int J Solids Struct 193, 222 (2020)
[7] J. F. Peters, M. Muthuswamy, J. Wibowo, A. Tordesillas, Phys Rev E 72, 041307 (2005)

[8] K. H. Roscoe, A. N. Schofield, C. P. Wroth, Géotechnique 8, 22 (1958)

[9] V. Šmilauer et al., Yade Documentation 2nd ed. The Yade Project. DOI 10.5281/zenodo.34073 (2015) (http://yade-dem.org/doc/)

[10] A. Tordesillas, D. M. Walker, Q. Lin, Phys Rev E 81, 011302 (2010)

[11] H. Zhu, H. N. G. Nguyen, F. Nicot, F. Darve, J Mech Phys Solids 95, 112 (2016) 